\title{
Field-Trial of a high-budget, filterless, $\lambda$-to-the-user, UDWDM-PON enabled by an innovative class of low-cost coherent transceivers
}

\author{
Marco Presi, Massimo Artiglia, Fabio Bottoni, Mario Rannello, Ivan Valdez-Cano, Jaison Tabares, Juan- \\ Camilo Velásquez, Saeed Gasemi, Victor Polo, Guang Yong Chu Student Member, IEEE, \\ Josep Prat, Member, IEEE, Gregorio Azcarate, Robert Pous, Chantal Vilá, \\ Helene Debregeas, Gemma Vall-llosera, Albert Rafel, and Ernesto Ciaramella, Senior Member, IEEE
}

\begin{abstract}
We experimentally demonstrate an innovative Ultra Dense Wavelength Division Multiplexing (UDWDM) Passive Optical Networks (PON) that implements the full $\lambda$-to-the-user concept in a filterless distribution network. Key element of the proposed system is a novel class of coherent transceivers, purposely developed with a non-conventional technical approach. Indeed, they are designed and realized to avoid D/A-A/D converter stages and Digital Signal Processing (DSP) in favor of simple analog processing so that they match system, cost and power consumption requirements of the access networks without sacrificing the overall performance. These coherent transceivers target different use case scenarios (residential, business, fixed, wireless) still keeping perfect compatibility and co-existence with legacy infrastructures installed to support gray, Time Division Multiplexed (TDM) PON systems. Moreover, the availability of coherent transceivers of different cost/performance ratios allows for deployments of different quality service grades. In this paper, we report the successful field trial of the proposed systems in a testbed where 14 UDWDM channels (and one legacy E-PON system) are transmitted simultaneously in a dark-fiber network deployed in the city of Pisa (Italy), delivering real-time and/or test traffic. The trial demonstrated filterless operations (each remote node selects individually its own UDWDM channel on a fine $6.25 \mathbf{G H z}$ grid), real-time GbE transmissions (by using either fully analog or light digital signal processing), multirate transmission ( 1.25 and $10 \mathrm{~Gb} / \mathbf{s} /)$, high Optical Distribution Network loss $(18 \div 40 \mathbf{d B})$ as well as a bidirectional channel monitoring system.
\end{abstract}

Index Terms-WDM networks, Access Networks, Passive Optical Networks, Coherent Detection, Homodyning, Heterodyning

M. Presi, M. Artiglia, M. Rannello, Fabio Bottoni and E. Ciaramella are with Scuola Superiore Sant'Anna, via G. Moruzzi, 156127 PISA (ITALY). E-mail: marco.presi@sssup.it

Fabio Bottoni is now with Oclaro, via ... Milano, ITALY email: fabio.bottoni@oclaro.com

I.V. Cano, J. Tabares, J. C. Velsquez, S. Ghasemi, V. Polo, G. Y. Chu and J. Prat are with Universitat Politecnica de Catalunya (UPC), c. Jordi Girona 1-3, 08034 Barcelona, Spain. E-mail ivan.cano@tsc.upc.edu

H. Debrgeas is with III-V Lab, joint laboratory of Nokia Bell Labs, Thales Research and Technology, and CEA Leti, Palaiseau, France. E-mail: Helene.Debregeas@3-5lab.fr

G. Vall-llosera is with Ericsson Research, Farogatan 6, 16480 Stockholm, Sweden. E-mail: gemma.vall-1losera@ericsson.com

R. Pous, G. Azcrate, and C. Vil are with Promax, c. Francesc Moragas 71, 08907 L'Hospitalet de Llobregat, Spain. E-mail: cvila@promax.es

A. Rafel is with BT Technology Service \& Operations, Adastral Park, Martlesham Heath, Ipswich, UK. E-mail: albert.2.rafel@bt.com

This work was partly supported by the European Commission FP7-ICT program under Grant 318515.

TThe authors would like to acknowledge the Municipality of Pisa (ITALY) and Agestel for having made available the city fiber system.

Manuscript received April 19, 2005; revised August 26, 2015.

\section{INTRODUCTION}

$\mathbf{T}$ HE recent standardization of the Next Generation Passive Optical Network 2 (NG-PON2) [1], the designated candidate technology upgrade of currently installed $\mathrm{B} / \mathrm{E} / \mathrm{G} / \mathrm{XG} / \mathrm{NG}$ PON systems, introduced for the first time, after almost a decade of research, the Wavelength Division Multiplexing (WDM) technology in optical access systems as an overlay to the Time Division Multiple Access (TDMA) signals [2]. However being an upgrade of gray Passive Optical Networkss (PONs) with Optical Distribution Network (ODN) realized only by power splitters and drop fiber trunks, WDM must be introduced by preserving the filterless character of deployed networks plant, i.e. without requiring the installation of optical filters in the ODN [3], [4]. NG-PON2 solved this issue by adopting low-cost tuneable optical thin-film filters inside the Optical Network Units (ONUs). Presently, these filters have usually large bandwidths, i.e. can provide only wavelength selection on $100 \mathrm{GHz}$ (or higher) grids [5]-[7]. On the other hand, upcoming network orchestration technologies favoring grid-less operation and/or featuring the to the user paradigm require transceivers capable of finer frequency selectivity [8]. Coherent transceivers [9] are today operated in optical core networks thanks to their capability of implementing sophisticated Digital Signal Processing (DSP), which allows for for compensation of transmission impairments [10], high spectral efficiency [11] and/or software-defined operations [12]. To attain these functionalities, DSP requires costly hardware (high bandwidth and high resolution Digital-to-Analog Converter (D/A) and Analog-to-Digital Converter (A/D) as well as highend processors and software units [13]-[15]. Furthermore, expensive optical devices (e.g. low-line-width lasers) and passive components (e.g. $90^{\circ}$ hybrid couplers) are also needed. All of these implementation features prevented the use of coherent detection in access networks so far, because cost requirements here are very much tight: it is indeed expected that PON equipment must have cost levels comparable with current consumer electronics, especially at user premises. However, many of the functionalities offered by present coherent systems are not required in the access network, where low data rate per user is typically expected (well below $10 \mathrm{~Gb} / \mathrm{s}$ ), the transmission reach does not exceed $100 \mathrm{~km}$ and Kerr-induced non-linear impairments are usually not triggered. 


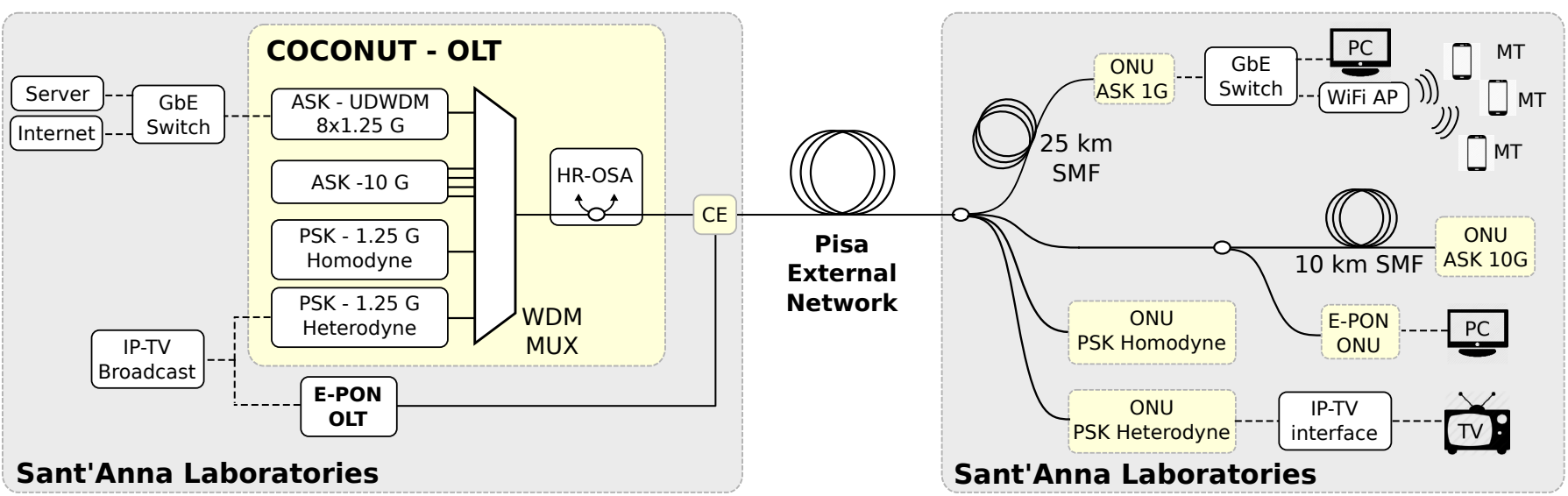

Figure 1. Schematic representation of the field trial setup. Gray areas represent the equipment placed in the lab. OLT: Optical Line Terminal; HR-OSA: HighResolution Optical Spectrum Analyser; CE: Co-existence Element; SMF: G.652 Single Mode Fiber; ONU: Optical Network Unit; PC: Personal Computer; AP: Access Point; MT: Mobile Terminal; TV: Television

Therefore the authors believe that coherent detection can play a significant role in the access network, but the corresponding design must be redefined and optimized for access requirements: this may allow to take advantage of some key features of coherent detection. These systems can have very low sensitivity (thus increasing the system power budget and the tolerable ODN insertion loss, which are very critical in the access domain); moreover, they allow for filter-less and colorless operations [8], [16], [17]. These considerations stimulated new research lines aiming at determining if coherent detection could be effectively revised in order to be implemented in the optical access network domain. Among the many different approaches considered, we decided to focus on the two aforementioned aspects: low-sensitivity and fine wavelength selectivity, by trading receiver simplicity for high-spectral efficiency and complex DSP functionalities. By focusing on these two parameters, we were able to design a new class of coherent transceivers featuring:

- use of low cost lasers (Distributed FeedBack laser (DFB) of pre-allocated wavelength) with coarse wavelength control rather than expensive low-line-width tuneable External Cavity Laser (ECL) [18]

- direct laser modulation analogue processing (or very light DSP in few selected cases) for reliable and low-latency real-time operation

- low-cost receiver front-end optical couplers (either $3 \times$ 3 or $2 \times 2$ fused fiber couplers) rather than costly $90^{\circ}$ hybrids [19], [20]

- wavelength reuse (when useful)

- low-cost, coherent-based monitoring system to inspect and manage the multi $\lambda$ system and the channel activation/deactivation [21]

This new class of coherent receivers was developed over the last three years within the COCONUT project [22], demonstrating a low power consumption level, and moderate cost per bit, if compared to other competitive coherent technologies [23], [24]. They thus show a potential for being effectively considered in real access network deployments. This new class of receivers has been developed initially to address the issue of upgrading legacy infrastructure the capabilities; yet, most of their features (channel selectivity at the receiver, flexible wavelength allocation, low receiver sensitivity) can be advantageously exploited in new deployments (the so called "green fields") or even exploited in alternative or novel ODNs designs (for example those that could be considered in FSAN). Here we provide a detailed description of the field trial, which was also open to public visitors, where these new transceivers have been put together showing the full capabilities of the proposed $\lambda$-to-the-user system on a real fiber network deployed in the city of Pisa (Italy), connected directly to/from Scuola Superiore Sant'Anna laboratories. The fiber plant has been made available by courtesy of Agestel (a local company). High-power budget (up to $40 \mathrm{~dB}$ ) filterless Ultra Dense Wavelength Division Multiplexing (UDWDM) operations (14 WDM channel with spacing as low as $6.25 \mathrm{GHz}$ ), different data rates $(1.25$ to $10 \mathrm{~Gb} / \mathrm{s})$, co-existence with legacy PON systems, real-time traffic delivery in different use case scenarios were demonstrated in eight concurrent experiments through the four flavors of COCONUT coherent transceivers. Although the performances of the single systems may have been already reported in reference of lab environments [25]-[27], we provide here an extended report of the first demonstration of their joint operation in a field trial condition, also showing for the first time real-time traffic operation, with Ethernetbased applications and high-definition video transmission. In addition coexistence with E-PON system is proven, in a typical evolution case. These results demonstrate for the first time that when all the systems are operated simultaneously in a real filterless network scenario, providing real service distribution their performances are not altered significantly, demonstrating the colorless property of the receivers, the resilience to their interaction and to the network reflections. This paper is organized as follows: in section II we provide a description of the trial network and introduce the five coherent transmission systems tested. In section III we summarize the testbed results, while in section IV we discuss the potential applications of the single transceivers to possible use cases. Finally in Section V we draw the conclusions. 


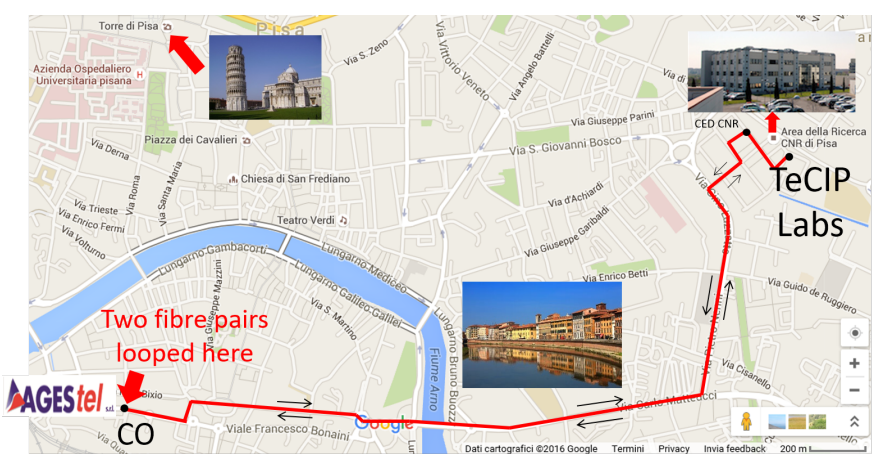

Figure 2. Map of the dark fiber deployment in loop configuration connecting the Scuola Superiore Sant'Anna laboratories (top right in the map) laboratories to the Pisa city center.

\section{Field TRial Description}

The setup of the field trial is schematically represented in Figure 1. At the Scuola Sant'Anna lab, we simulated a central office hosting two Optical Line Terminal (OLT) systems: the COCONUT OLT and a commercially available E-PON. A co-existence WDM filter combined the two OLTs. The COCONUT OLT consisted of four different groups of transceivers, based on different modulation formats (either Amplitude Shift Keying (ASK) or Phase Shift Keying (PSK)), modulation types (direct or external), detection schemes (heterodyne or intradyne) and bit-rate (1.25 or $10 \mathrm{~Gb} / \mathrm{s})$. We used an E-PON system because of availability of the equipment, but this is perfectly equivalent to a G-PON, since E-PON and G-PON share the same wavelength plan and they differ mainly for the framing, so from co-existence point of view this test is equivalent of testing a G-PON.

We also emulated a scenario where asymmetrical bit-rates are used (e.g. $10 \mathrm{~Gb} / \mathrm{s}$ downstream and $1.25 \mathrm{~Gb} / \mathrm{s}$ upstream). Similarly, different experiments used three different wavelength allocation plans for the Upstream (US)/Downstream (DS) transmission: ultra-narrow spacing $(2.5 \mathrm{GHz})$, UDWDM spacing $(6.25 \mathrm{GHz})$ and large spacing $(100 \mathrm{GHz}$ ITU grid). This allowed to emulate different field deployments (green or brown field). In all cases, a cascade of power splitters and a WDM multiplexer combined these signals [28]. The WDM Multiplexer (MUX) had $100 \mathrm{GHz}$ channel spacing and $100 \mathrm{GHz}$ Full Width at Half Maximum (FWHM) on each channel. For UDWDM operations, up to 8 channels fitted one MUX port on a $6.25 \mathrm{GHz}$ grid. The channel monitoring system, provided by a low-cost High-Resolution Optical Spectrum Analyser (HR-OSA), was placed at the MUX output through an optical by-pass allowing the monitoring of both upstream and downstream traffic. The combined OLT output was then directly connected to an external feeder fiber. The feeder is composed of dark fibers that are part of an operating fiber network deployed in the city of Pisa: it was made available by a Agestel, and was composed by a series of dark G.652 Single Mode Fiber (SMF) fiber trunks in a loop configuration from Sant'Anna facilities to downtown and back, as shown in Figure 2. The loop length was approximately $10 \mathrm{~km}$. This external section included several connections
Table I

WAVELEngth Allocation Plan

\begin{tabular}{cccc}
\hline System & Link & Frequency (THz) & Wavelength $(\mathrm{nm})$ \\
\hline ASK Intradyne & US & $\underline{193.0}$ & $\underline{1551.72}$ \\
PSK Homodyne & US/DS & 193.2 & 1550.92 \\
ASK $4 \times 10 \mathrm{~Gb} / \mathrm{s}$ & DS & $194.3-193.7$ & $1550.12-1547.12$ \\
DPSK $\underline{\text { Heterodyne }}$ & US/DS & 193.5 & 1549.32 \\
ASK Intradyne & DS & 193.9 & 1546.12 \\
& & $(8 \times 1.25 \mathrm{~Gb} / \mathrm{s})$ & \\
\hline
\end{tabular}

(SC/PC connectors) in outside cabinets, with a total insertion loss of $10 \mathrm{~dB}$. The end of this fiber loop reentered the lab and connected to a drop fiber system realized by a cascade of power splitters and fiber spools to reach five ONUs, each one communicating with its own corresponding transceiver at the OLT side (either E-PON or the COCONUT ones). The full ODN had a maximum reach of $35 \mathrm{~km}$, a maximum loss of $40 \mathrm{~dB}$ and ONUs maximum differential loss of $22 \mathrm{~dB}$. We note that the maximum differential and absolute loss values exceeded by 7 and $5 \mathrm{~dB}$, respectively, those recommended in current PON standards [29]. For the sake of the simplicity, each communication system used its own wavelength band. The exact wavelength allocation is reported in Table I. As will be seen, some systems had a very low frequency separation between downstream and upstream signal, which allowed to allocate both into a single ITU-T WDM frequency slot; other systems were configured so that two different ITU-T slots were used for upstream and downstream. Both at the OLT and the ONU sides, some of the transceivers have been connected to Ethernet switches or multimedia streaming servers to demonstrate network operations in a heterogeneous distribution of real-time traffic. In the following sections, those coherent systems will be reviewed in detail.

\section{A. UD-WDM $8 \times 1.25 \mathrm{~Gb} / \mathrm{s}$ ASK Intradyne Real-Time analog system}

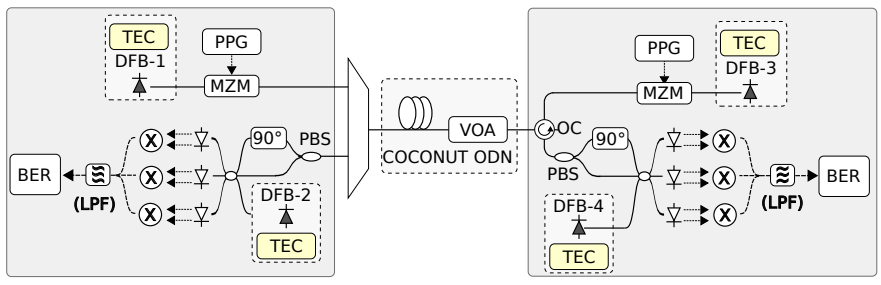

Figure 3. ASK system schematics. PPG: Pulse Pattern Generator; TEC: Thermo-Electric Cooler; MZM: Mach-Zendher Modulator; PBS: Polarization Beam Splitter; LPF: Low-Pass Filter; OC: Optical Circulator; ODN: Optical Distribution Network

We setup a bidirectional $1.25 \mathrm{~Gb} / \mathrm{s}$ ASK system, which makes use of two identical transceivers exploiting two different wavelength bands to realize simultaneous upstream and downstream transmissions. This is achieved by using two distinct Arrayed Waveguide Router (AWG) ports at the OLT side (one for downstream and one for the upstream), and an optical circulator at the ONU side (see Figure 3). The transceivers 
comprise a transmitter based on external modulation (due to the limited number of available directly modulated lasers) driven either by a Pulse Pattern Generator (PPG) for Bit Error Ratio (BER) measurements or by a Gigabit Ethernet $(\mathrm{GbE})$ interface. Both transmitters use commercial DFB lasers. The receivers use a polarization independent scheme based on intradyne operation [30] and featuring a $3 \times 3$ optical hybrid coupler [19]. The received signal undergoes first in a polarization split and rotate stage. After this, its two orthogonal polarization components are injected in the $3 \times 3$ coupler together with the output of the Local Oscillator (LO), which is also a DFB laser. As a result of the first stage, all these three fields enter the coupler with same State of Polarization (SoP). The local oscillator is tuned to obtain proper intradyne regime (detuning between signal and LO around $80 \%$ of the bit-rate, $900 \mathrm{MHz}$ in this case) [30]. The coupler output lights are then detected by three PIN photodiodes. These three photocurrents are squared by using off the shelf analog multipliers, added and then low-pass filtered ( $4^{\text {th }}$ order Bessel, $933 \mathrm{MHz}$ bandwidth) to yield the recovered ASK signal [31]. The analog processing stage output is directly connected either to a BER tester or to a $\mathrm{GbE}$ module in case of real-time application demos. UDWDM operation has been also tested, by multiplexing 8 downstream ASK $1.25 \mathrm{~Gb} / \mathrm{s}$ channels onto a single AWG input port at the OLT as shown in Figure 4. The two setups differ mainly in the presence of the 8 downstream channels which are first grouped by using a cascade of power splitters and then coupled into a single $\mathrm{AWG}$ port. Since the AWG has a pass-band of $50 \mathrm{GHz}$ and Gaussian shape, suitable pre emphasis was used to make the channels, spaced by $6.25 \mathrm{GHz}$, leave the AWG with a uniform power level (about $3 \mathrm{dBm} /$ channel, see also Figure 8 ). Due to the lack of 8 independent PPGs we modulated the channels at groups of four, dividing them in odd and even combs driven by PRBS sequences of different lengths $\left(2^{15}-1\right.$ and $\left.2^{31}-1\right)$ produced by two independent PPGs, so that adjacent channels are fully uncorrelated. At the ONU side, the LO was thermally tuned to receive the selected individual channel and measure its performance.

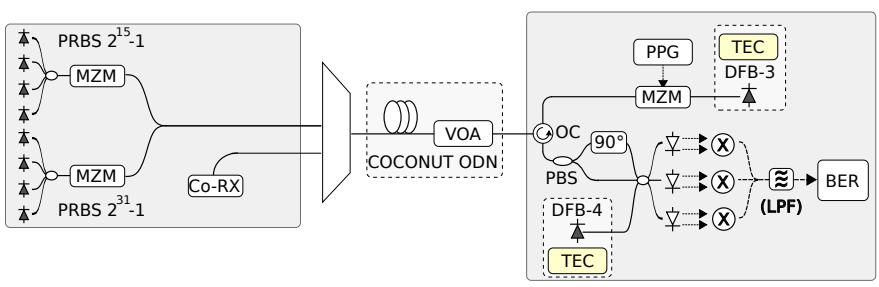

Figure 4. UDWDM experimental setup. MZM: Mach-Zendher Modulator; OC: Optical Circulator; PBS: Polarization Beam Splitter; PPG: Pulse Pattern Generator; TEC: Thermo-Electric Cooler; LPF: Low-Pass Filter; ODN: Optical Distribution Network; VOA: Variable Optical Attenuator

\section{B. $4 x 10 \mathrm{~Gb} / \mathrm{s}$ ASK Intradyne system}

This system used $4 \times 10 \mathrm{Gbit} / \mathrm{s}$ directly-modulated lasers (Directly Modulated DFB Laser (DML)), placed on a $100 \mathrm{GHz}$ grid with $\lambda$ between 1550.12 and $1547.12 \mathrm{~nm}$. These lasers were modulated by 4 PRBS streams obtained by exploiting the direct and inverted outputs of two independent PPGs (peakto-peak modulation around 1.3 Volt). At the ONU, we used a

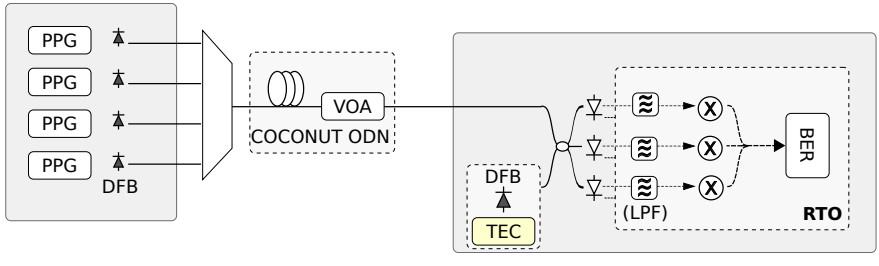

Figure 5. $4 \times 10 \mathrm{~Gb} / \mathrm{s}$ System Setup. LPF: Low-Pass Filter;TEC: ThermoElectric Cooler; ODN: Optical Distribution Network; VOA: Variable Optical Attenuator

version of the ASK receiver, which was polarization dependent because of the limited number of available components, and was realized by using only a $3 \times 3$ optical coupler, a DFB as LO and 3 PIN photodiodes ( $15 \mathrm{GHz}$ bandwidth). The LO wavelength was tuned to receive each channel (with a signal-LO detuing at about $3 \mathrm{GHz}$ ), while its polarization was manually controlled. Polarization independent operation of the receiver has been recently reported [32]. The signal processing was realized offline, using a real-time oscilloscope (Real Time Oscilloscope (RTO), $13 \mathrm{GHz}$ analog bandwidth, $40 \mathrm{GSa} / \mathrm{s}$ ): it emulated 3 parallel processing stages, each having a $3 \mathrm{GHz}$ low-pass filter (for frequency-to-amplitude conversion) and a squaring function: the three processed signals were then added to recover the ASK signal. BER of the received signal was also computed within the RTO by a custom developed routine [33].

\section{C. $1.25 \mathrm{~Gb} / \mathrm{s}$ DPSK heterodyne Real-Time analog system}

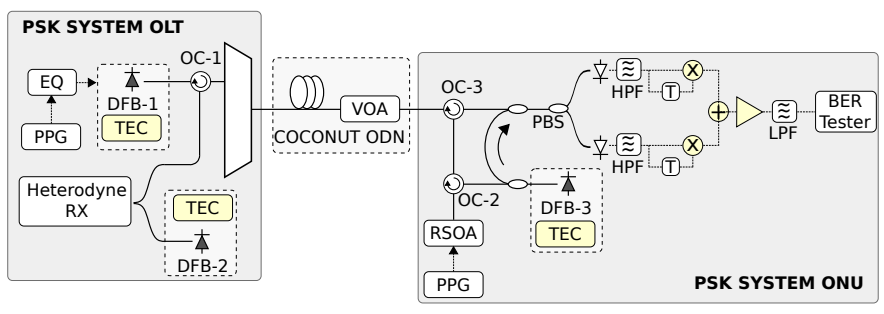

Figure 6. PSK system setup. PPG: Pulse Pattern Generator; OC: Optical Circulator; TEC: Thermo-Electric Cooler; EQ: Equalizer; LPF: Low-Pass Filter; R-SOA: Reflective Semiconductor Optical Amplifier; PBS: Polarization Beam Splitter; HPF: High-Pass Filter; BERT: Bit Error Ratio Tester

This system exploits a heterodyne transceiver whose detailed scheme is depicted in Figure 6. At the OLT side, the transmitter consisted of a pre-equalized DML (DFB-1), which produced a phase-modulated signal [26]. It was driven either by a PPG or from a GbE interface. The DFB was temperature controlled and emitted at $1549.32 \mathrm{~nm}$. A circulator separated the down/upstream directions before being multiplexed by the WDM MUX. The downstream signal reached the COCONUT ODN with $0 \mathrm{dBm}$ optical power. At the ONU side, the receiver consisted in a polarization independent heterodyne mixer [26]. The ONU front-end used a network of optical circulator and $3 \mathrm{~dB}$ couplers/splitters separating US/DS traffic and allowing to use a single DFB laser (DFB-3 in Figure 6) simultaneously as LO and seed for the upstream Reflective Semiconductor Optical Amplifier (R-SOA) transmitter [34] [35]. The LO emission frequency was shifted by $+2.5 \mathrm{GHz}$ from the receiving signal and its power was set to $0 \mathrm{dBm}$. The two polarization diversity branches consisted in a single ended 
photodiodes ( $7 \mathrm{GHz}$ E/O bandwidth) followed by a high-pass filter of $1.2 \mathrm{GHz}$ cutoff frequency and an analogue delay and multiply circuit to decode the signal. The two branches were finally combined, low-pass filtered at $1 \mathrm{GHz}$, amplified and sent to the Clock and Data Recovery (CDR) circuit for BER measurements performed by a common Bit Error Ratio Tester (BERT). The US transmitter used a directly-modulated TOCAN R-SOA, biased at $70 \mathrm{~mA}$ with $18 \mathrm{~dB}$ linear gain although here under saturation for phase modulation, and was coupled to the ODN with $3 \mathrm{dBm}$ optical power. At the OLT, the US signal was detected by a polarization dependent coherent heterodyne receiver with another DFB laser (DFB-2) as LO, which implemented the same analogue processing described above but using balanced detection [34].

\section{D. $1.25 \mathrm{~Gb} / \mathrm{s}$ PSK Homodyne Real-Time digital system}

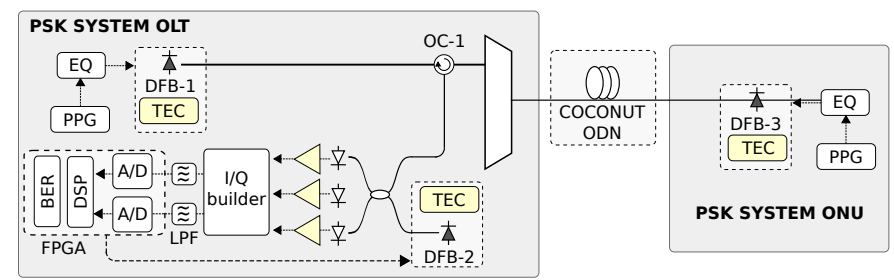

Figure 7. PSK Homodyne system setup. PPG: Pulse Pattern Generator; OC: Optical Circulator; TEC: Thermo-Electric Cooler; EQ: Equalizer; LPF: LowPass Filter; A/D: Analog-to-Digital Converter; DSP: Digital Signal Processing; ODN: Optical Distribution Network

$1.25 \mathrm{~Gb} / \mathrm{s}$ homodyne PSK system was also implemented by using directly phase-modulated DFB lasers (see Figure 7). However, in this case the OLT upstream receiver exploited a dedicated FPGA platform for real time detection. The ONU upstream transmitter used also a directly phase-modulated laser (DFB-3), whose line-width was $\Delta \nu=4 \mathrm{MHz}$ and emitted at $1550.9 \mathrm{~nm}$ (see table I). At the OLT, a digital coherent receiver comprised a $3 \times 3$ optical hybrid coupler to mix the incoming signal with the LO (DFB-2), emitting $3 \mathrm{dBm}$ output power. The State of Polarization (SOP) was manually optimized at the receiver input. The three output signals reached three single-ended TO-CAN packaged photodetectors $(\mathrm{BW}=2 \mathrm{GHz})$. The electrical signals were then amplified and properly combined to obtain the in-phase (I) and quadrature (Q) components, which were then low-pass filtered at $1 \mathrm{GHz}$ by two micro strip Bessel filters for anti-aliasing and noise rejection. Then, each electrical signal was A/D converted at the Nyquist rate of $2.5 \mathrm{GSa} / \mathrm{s}$ at 8 vertical bits and sent to a FPGA unit (Xilinx Virtex-6) for real time processing. The digital signal processing (DSP included a de-skew stage for channel alignment and a data demodulation stage using two samples per symbol. In addition, a frequency estimation stage generated an error signal driving continuously the LO (DFB2) to match its frequency with the frequency of the incoming signal, with an error below $\pm 50 \mathrm{MHz}$ [36]. Such an error signal was D/A converted by a 1-bit serial converter based on pulse width modulation and sent to the LO control circuit for thermal and current tuning. Finally, data decision was performed by selecting the best sample for each bit and the
BER was measured inside the FPGA by direct error counting on the recovered data sequence.

\section{E. High-Resolution Optical Spectrum Analyser}

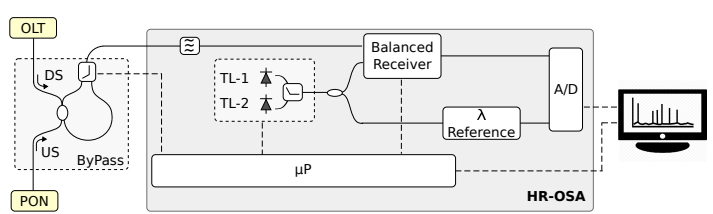

Figure 8. HR-OSA setup. OLT: Optical Line Terminal; DS: Downstream; US: Upstream; A/D: Analog-to-Digital Converter; PON: Passive Optical Networks

The monitoring equipment exploited a high-resolution optical spectrum analyzer (HR-OSA), which is able to detect individual channels also when operating on the $6.25 \mathrm{GHz}$ UDWDM grid. Indeed, the HR-OSA has a resolution bandwidth as low as $100 \mathrm{MHz}$. This HR-OSA was developed adhoc to test the COCONUT system. It is based on coherentdetection and its structure is shown in Figure 8. Each tuneable laser scans a $5 \mathrm{~nm}$ window in the C-band. By stacking more tuneable lasers, each one covering $5 \mathrm{~nm}$, it is possible to widen the analysis band. The HR-OSA also provides a management user interface able to monitor all the active channels in the network, either in upstream or downstream, and to identify active transmitters (located either in ONUs or at the OLT side). The identification system is meant not only to monitor the health status of the active channels, but also to assist new ONU activations (i.e. new lasers to be turned on), by contributing with detailed information about the network status. Bidirectional monitoring is achieved by tapping $1 \mathrm{~dB}$ power at the OLT ingress/egress port.
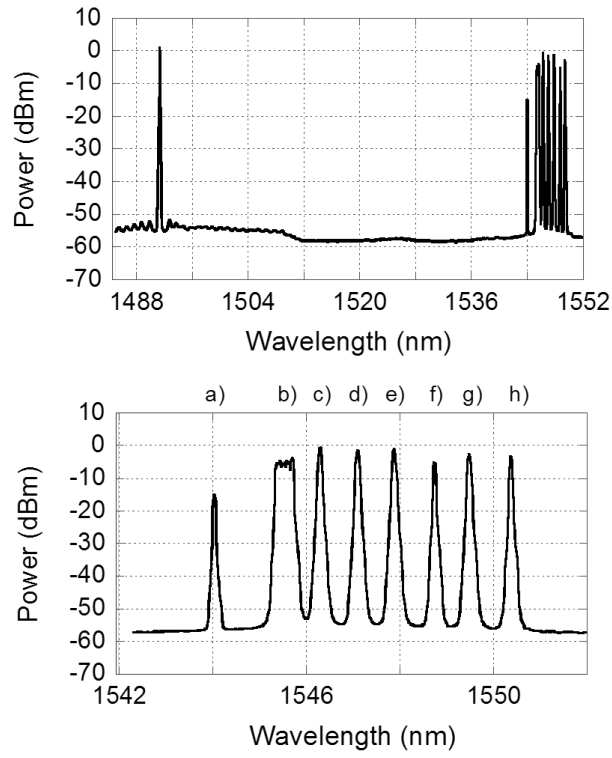

Figure 9. Top: optical spectrum of downstream channels; Bottom: C-band COCONUT channels. a) pilot; b) $8 \times 1.25 \mathrm{~Gb} / \mathrm{s}$ ASK; c-e) $10 \mathrm{~Gb} / \mathrm{s} \mathrm{ASK}$; f) $1.25 \mathrm{~Gb} / \mathrm{s}$ DPSK heterodyne; g) $10 \mathrm{~Gb} / \mathrm{s} \mathrm{ASK}$; h) $1.25 \mathrm{~Gb} / \mathrm{s}$ DPSK homodyne 


\section{System PERformanCE}

We now report the final trial results of the systems illustrated in the previous sections. Figure 9 (top) reports the optical spectrum of the 14 downstream channels spanning from 1490 to $1552 \mathrm{~nm}$. The bottom spectrum represents a magnification of the band occupied by the COCONUT channels (see also Table I for the detailed channel allocation).

\section{A. $1.25 \mathrm{~Gb} / \mathrm{s}$ ASK systems}
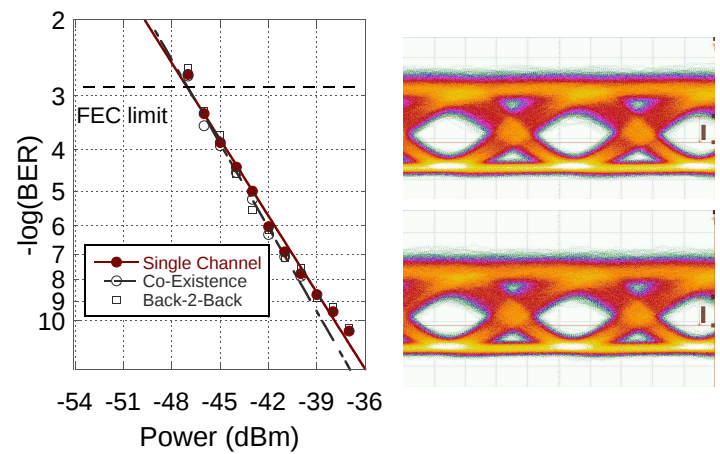

Power $(\mathrm{dBm})$
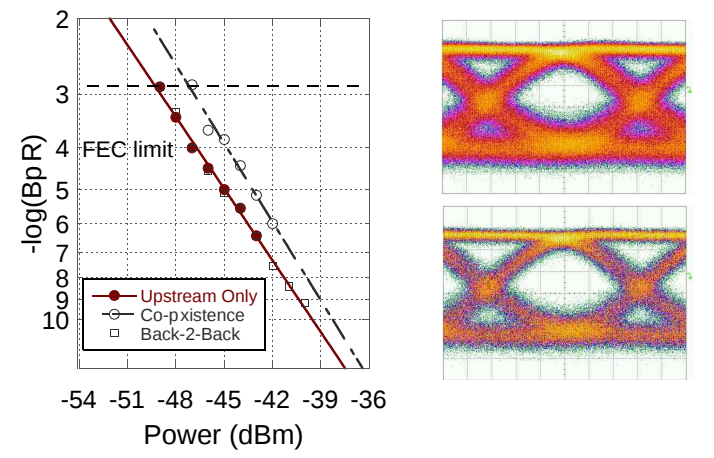

Figure 10. ASK system performance. BER Measurements and eye diagrams recorded for the downstream (top row) and upstream(bottom row)

The ASK system performances are shown for both downstream and upstream directions in Figure 10. This system also shows error-free operation without apparent BER floors. In the two directions, the sensitivities are slightly different $\left(-48\right.$ and $-47 \mathrm{dBm}$ at BER $=10^{-3}$, respectively), although the down/upstream receivers were nominally identical. We note that the system performances were not altered by the presence of the other 13 channels in the network: the absence of appreciable power penalty observed proves that the receiver is tolerant to cross-talk induced by these other channels. However, we noticed a non-negligible power penalty in the upstream direction, but this was observed only when the PSK ONU transmitter was turned on. This power penalty is clearly due to the wide-band ASE noise of the R-SOA in the PSK upstream transmitter, which reduced the Optical Signal-to-Noise Ratio (OSNR) of the received signal down to about $20 \mathrm{~dB}$. This reduction of OSNR can be clearly seen in the spectra reported in Figure 11.

This effect was particularly evident when the ASK and PSK ONUs had a large differential loss (the PSK ONU was closer to the OLT so that its optical power to the RX was higher than the received ASK signal). The UDWDM system
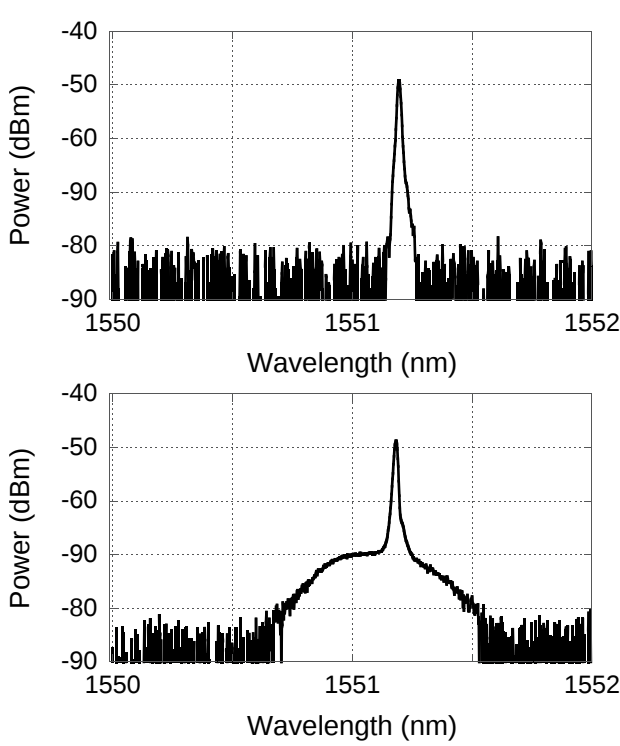

Figure 11. Optical spectrum of the upstream ASK signal when the PSK ONU is turned OFF (top) or ON (bottom)

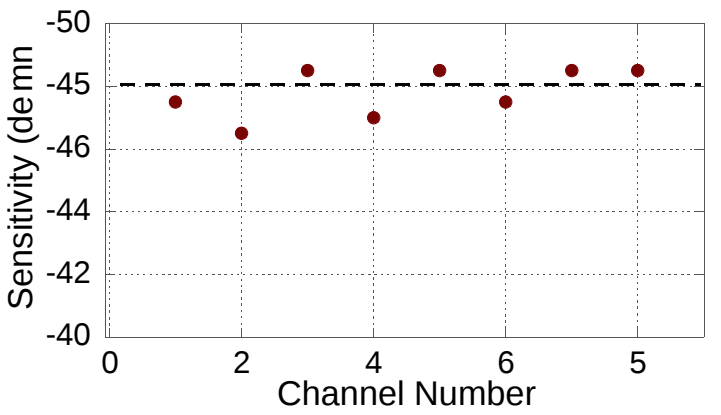

Figure 12. Average BER measured at the FEC level $\left(1.8 \times 10^{-3}\right)$ for the 8 ASK channels.

was then tested in the downstream direction: we measured all the 8 UDWDM channels by tuning the ONU LO frequency to select them individually. The measurements confirmed that all WDM channels had homogeneous performance. We report in Figure 12 the sensitivity values of the 8 channels measured at the FEC level. As can be seen, the channels shown a FEC level sensitivity of about $-48 \mathrm{dBm}$ : the small difference is attributed to emission power fluctuations of the 8 transmitters (laser power stability is about $1 \mathrm{~dB}$ over few hours for the local oscillator laser used in this experiment). UDWDM operations benefit from the availability of the highresolution optical spectrum analyser, which drives the network monitoring system.

In Figure 13 we report the detailed optical spectrum of the UDWDM system, recorded with a Resolution BandWidth (RBW) of $100 \mathrm{MHz}$. As can be seen, not only the 8 UDWDM channels are well distinguished, but also their modulation spectrum can be clearly observed. The channels here appear not uniform in power, since they have a scrambled polarization, while the OSA prototype is not polarization independent. In the bottom inset of the spectrum analyser screen, we can see also the monitoring function: it shows a miniaturized spectrum of active channels in each ITU-T frequency band. 


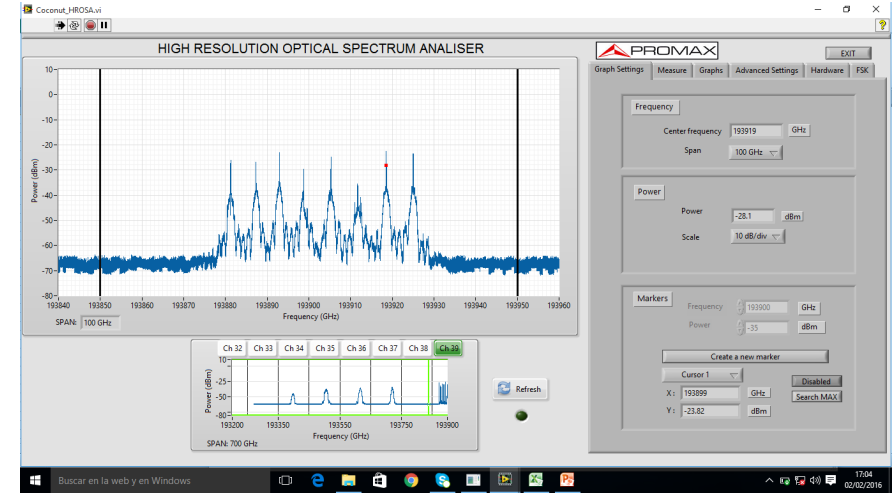

Figure 13. High Resolution Optical Spectrum of the 8 channel UDWDM system. In this example, The HR-OSA is set to show a $100 \mathrm{GHz}$ span with a RBW $100 \mathrm{MHz}$ interface allows to select frequency bands corresponding to different ITU-T channels.

\section{B. DPSK Systems}

The DPSK system performance is reported in Figure 14. As can be seen, the receiver sensitivities at $\mathrm{BER}=10^{-3}$ and $\mathrm{BER}=10^{-9}$ were -37 and $-31.5 \mathrm{dBm}$ respectively. No BER floor appeared in the considered range of optical power levels. In the same figure insets, we see the eye diagrams recorded at $\mathrm{BER}=10^{-5}$. For comparison, the back-to-back line was also added, showing a very negligible power penalty arising when all the COCONUT channels are activated in respect to the case in which only the PSK system operates. The effect of in-band Rayleigh Backscattering (RB) was limited because the DS and US signal carriers were frequency shifted by 2.5 $\mathrm{GHz}$, so that the back-reflections spectrum falls outside of the received signal bandwidth, as shown in 16 , so that their contribution is easily filtered out.

The PSK homodyne system featured the best sensitivity (around $-53 \mathrm{dBm}$ at a FEC level of $10^{-3}$ ) in back-to-back. Figure 15 shows the receiver performance and two sample eye diagrams, recorded at BER $10^{-9}$ and $10^{-3}$ in its polarizationdependent configuration. As can be seen after propagation across the network, the performance is not altered significantly when the signal is transmitted in upstream with the other COCONUT channels. However, a small power penalty (about

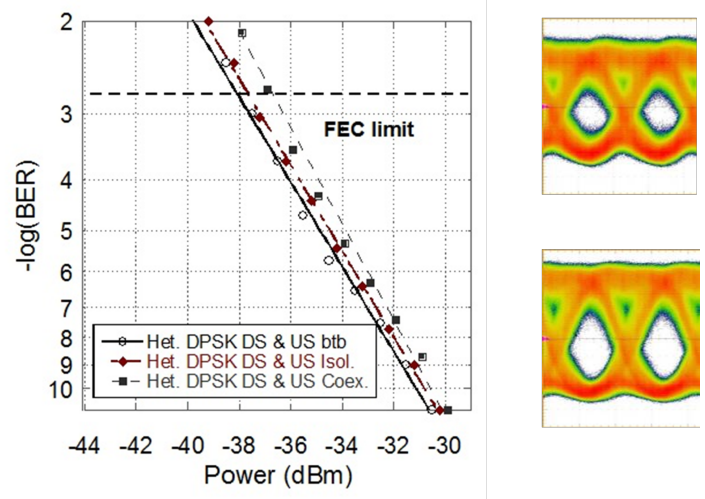

Figure 14. Downstream DPSK system performance. Eye diagrams refer to the back-to-back (bottom) and transmission (top) cases.
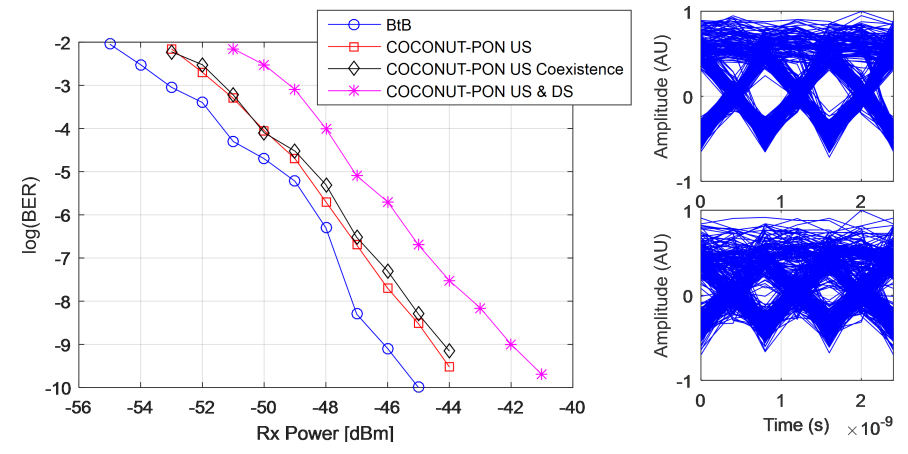

Figure 15. Upstream PSK system performance.

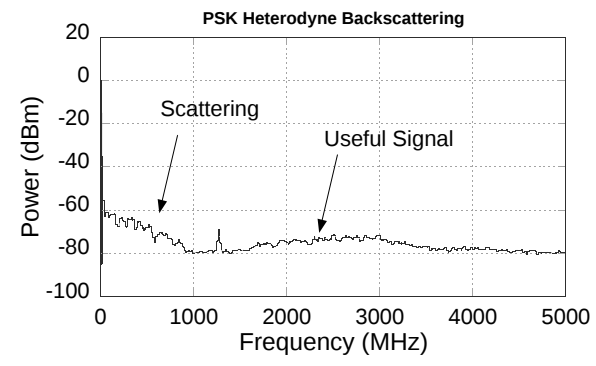

Figure 16. Electrical spectrum recorded after heterodyne beating in the DPSK

$4 \mathrm{~dB}$ ) appears in case of bidirectional transmission with US/DS channel separation of $6.25 \mathrm{GHz}$ : in this case, the reflection of the counter-propagating channels are aliased into the signal bandwidth. The use of photodiodes of narrower bandwidth and/or a higher frequency sample rate may alleviate this penalty. All the above real-time systems were equipped with a GbE interface, which made possible the end-to-end transmission of real-time traffic across the ODN, including streaming of HD video services (up to $4 \mathrm{~K}$ ) through the IPTV or raw protocols, high-speed data transfers and the delivery of wireless traffic to mobile users attending the demo. We note that for real traffic transmission, all the system demonstrators have been operated in the error-free regime (i.e., hardFEC coder/decoder were not implemented in favour of lowlatency operations) to overcome the lack of error correction capabilities in GbE transmissions. In all cases, stable end to end transfer rates exceeding $100 \mathrm{MB} / \mathrm{s}$ have been obtained by direct measurement of the throughput on the destination terminals and by using $\mathrm{GbE}$ analysers.

\section{C. $4 x 10 \mathrm{~Gb} / \mathrm{s}$ System}

$4 \times 10 \mathrm{~Gb} / \mathrm{s}$ operation was also assessed over the field-trial system, allowing to test its resiliency to the cross-talk generated by numerous COCONUT channel active in the testbed. We first measured the $4 \times 10 \mathrm{~Gb} / \mathrm{s}$ system performance over the ODN, first without the additional COCONUT channels, and then with all of them, (Figure 17-a and b respectively) in the same way as we did for the other experiments. While the propagation over the ODN did not introduce any significant power penalty in respect to the back to back, when all the 14 COCONUT channels are on, an acceptable power penalty of 

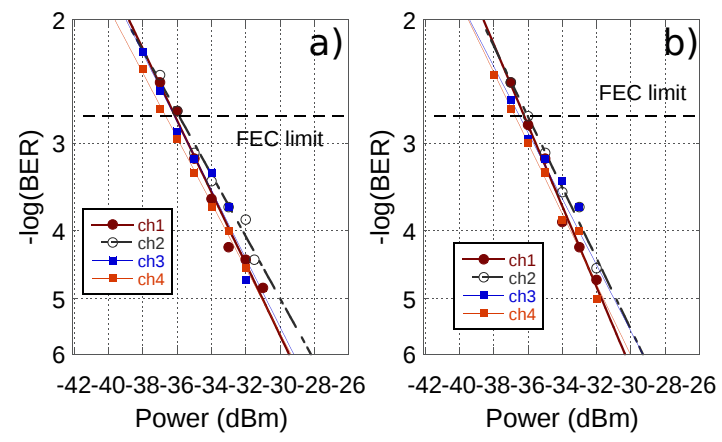

Figure 17. $4 \times 10 \mathrm{~Gb} / \mathrm{s}$ DML system performance across the ODN without (a) and with (b) COCONUT co-existing channels

about $1 \mathrm{~dB}$ at a BER of $10^{-5}$ can be observed. This result is well in-line with previous reports where the $4 \times 10 \mathrm{~Gb} / \mathrm{s}$ was tested alone [37]. This confirms that filterless ODN operation is still feasible also in the case of $10 \mathrm{~Gb} / \mathrm{s}$ operations. Since the receiver sensitivity is $-36 \mathrm{dBm}$, at FEC level, an overall power budget of about $40 \mathrm{~dB}$ can be achieved, considering a launch power of $10 \mathrm{dBm}$. Although it could be possible to use DSP to suppress numerically the interference due to the directdetection terms [19], we stress that in our implementation, the off-line signal processing did not include any complex stage dedicated to the suppression of the direct-detection terms. Assessing the maximum number of channels that can be handled by the receivers is currently under investigation.

\section{DISCUSSION}

The different coherent transceiver solutions that have been presented differ in modulation format (PSK, DPSK, ASK), bit rate $(1.25$ and $10 \mathrm{~Gb} / \mathrm{s})$, and detection techniques (homodyne, intradyne, heterodyne). While all of them address the need of low-cost and simplicity, low sensitivity and fine wavelength selectivity, each solution shows its peculiar features (summarized in Table II) that can make it specifically suitable for different use cases/scenarios originally identified in the COCONUT $\lambda$-to-the-user reference architecture [27]. To guarantee compatibility with existing infrastructures, COCONUT architecture targets an overlay of legacy single channel TDM Access PON Point-to-Multipoint systems and of coherent WDM PON Point-to-Point systems serving residential and customers services (FTTH) as well as mobile network cells and Wi-Fi access points (FTTCell), all operating over the same filterless ODN based on passive splitters. In this scenario the ASK $N \times 1.25 \mathrm{~Gb} / \mathrm{s}$ intradyne system, with its ultra-dense channel spacing, is particularly suitable for providing $1 \mathrm{~Gb} / \mathrm{s}$ FTTH to high residential user density areas where a high number of channels must fit in the limited available spectrum (brown-field). Its low sensitivity can be exploited to find the best trade-off between medium-long reach and split ratio level. Considering its sensitivity, the PSK homodyne receiver could be efficiently exploited in long-reach applications and PONs with very high split-ratios, allowing for example a further splitting level for serving a group of mobile micro-cells (FTTCell). Moreover it offers the best flexibility in green field deployments. The $10 \mathrm{~Gb} / \mathrm{s}$ coherent WDM system shows to
Table II

Use CASES AND AVAILABle Technology

\begin{tabular}{|c|c|c|}
\hline System & Main Feature & Use Case \\
\hline $\begin{array}{c}A S K \\
8 x 1.25 \mathrm{~Gb} / \mathrm{s} \\
\text { Intradyne }\end{array}$ & $\begin{array}{l}\text { UDWDM operation } \\
\text { and low sensitivity }\end{array}$ & $\begin{array}{l}\text { Brown-field with low } \\
\text { spectrum availability, } \\
\text { medium-long reach }\end{array}$ \\
\hline $\begin{array}{c}\text { PSK } \\
\text { Homodyne }\end{array}$ & Very low sensitivity & $\begin{array}{l}\text { Long-reach or green } \\
\text { field deployment }\end{array}$ \\
\hline $\begin{array}{c}A S K \\
4 x 10 \mathrm{~Gb} / \mathrm{s}\end{array}$ & Highest capacity/ $\lambda$ & $\begin{array}{l}\text { Business services, } \\
\text { radio access X-haul }\end{array}$ \\
\hline$\frac{\text { DPSK }}{\text { Heterodyne }}$ & Cost-effectiveness & $\begin{array}{l}\text { Short-reach on legacy } \\
\text { infrastructures }\end{array}$ \\
\hline
\end{tabular}

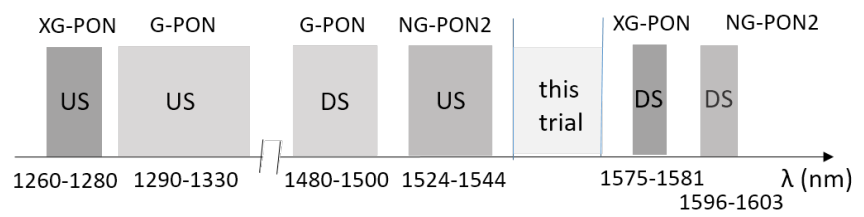

Figure 18. Wavelength allocation plan used in this field trial to ensure compatibility with other PON systems

be a good match for high speed point-to-point dedicated links such as business connections, or for radio access macro-cells (FTTCell).

The DPSK solution, finally, features the highest power efficiency and lowest estimated cost, since it involves only one laser at the ONU side, and directly modulated lasers at the OLT side, although it trades-off in terms of sensitivity. It addresses the needs of simplicity and low-cost of the architecture, and then it matches the requirements for upgrading legacy networks progressively adding dedicated $1 \mathrm{Gbit} / \mathrm{s}$ user connection in coexistence with G-PON or E-PON systems. As mentioned in the testbed description, the COCONUT system has been demonstrated over a deployed fibre network where we also operated a commercially available E-PON system (comprising both an OLT and an ONU): the system transmitted real IP traffic, generated by a local IP-TV broadcasting system.

We remark that in order to guarantee the co-existence, the frequency selective COCONUT transceivers were not equipped with any filter to reject E PON signals. On the other hand, a commercially available Co-Existence optical filter originally designed for NG-PON2 applications has guaranteed error-free E-PON operations: in other words, this demonstrated that the COCONUT system could co-exist with legacy PON systems without interfering or impairing them. Moreover we note that the wavelengths used in our demonstrator do not overlap with G/XG-PON and NG-PON2 wavelength allocation plans (see Figure 18). More generally, the proposed system can be flexible and conveniently adapted to work in any spectral range within the $\mathrm{C}$-band. The technology described here, due to its flexibility and high channel density, can therefore be particularly advantageous in upgrading existing ODNs where spectral availability is limited due to legacy equipment still in operation. On the contrary, in green field deployments, it could unleash the potential of an efficient spectrum usage thanks to the use of UDWDM. 


\section{CONCLUSION}

We experimentally demonstrated a field-trial access network where a new class of coherent transceivers implement the $\lambda$-to-the-user concept allowing real-time delivering of 1.25 and $10 \mathrm{~Gb} / \mathrm{s}$ in a filter-less optical distribution network. The network had losses up to $40 \mathrm{~dB}$ and included dark fibers deployed in Pisa, Italy. We have proven successful delivery of real-time Ethernet traffic, over the COCONUT interfaces, to both fixed and wireless access-points. The different transceivers developed for this system have different cost/performance specifications, making each of them suitable for the distribution of dedicated services or for different use cases connectivity. The proposed system is not only fully compliant with legacy optical distribution networks but can also share the network with legacy systems (E-PON in the demonstration). We therefore believe that the proposed system makes possible a smooth upgrade of legacy infrastructures without the need of any modification and allows extending the network capacity in terms of number of served users (up to 256 ) and reach (up to $100 \mathrm{~km}$ ).

\section{REFERENCES}

[1] "40-Gigabit Capable Passive Optical Networks (NG-PON2)", ITU-T Series Recommendations G.989.

[2] Y. Luo, X. Zhou, F. Effenberger, X. Yan, G. Peng, Y. Qian, and Y. Ma, "Time-and wavelength-division multiplexed passive optical network (TWDM-PON) for next-generation PON stage 2 (NG-PON2)," Journal of Lightwave Technology, vol. 31, no. 4, pp. 587-593, 2013.

[3] "Full Service Access Network (FSAN)." [Online]. Available: "http: //www.fsan.org"

[4] P. Chanclou, A. Cui, F. Geilhardt, H. Nakamura, and D. Nesset, "Network operator requirements for the next generation of optical access networks," IEEE Network, vol. 26, no. 2, 2012.

[5] D. Nesset, "NG-PON2 technology and standards," Journal of Lightwave Technology, vol. 33, no. 5, pp. 1136-1143, 2015.

[6] J.-i. Kani, F. Bourgart, A. Cui, A. Rafel, M. Campbell, R. Davey, and S. Rodrigues, "Next-generation PON-part I: Technology roadmap and general requirements," IEEE Communications Magazine, vol. 47, no. 11, 2009.

[7] R. Murano, "Optical component technology options for NGPON2 systems," in Optical Fiber Communication Conference. Optical Society of America, 2014, pp. M3I-1.

[8] J. Prat, I. N. Cano, M. Presi, I. Tomkos, D. Klonidis, G. Vall-llosera, R. Brenot, R. Pous, G. Papastergiou, A. Rafel et al., "Technologies for Cost-Effective udWDM-PONs," Journal of Lightwave Technology, vol. 34, no. 2, pp. 783-791, 2016.

[9] E. Ip, A. P. T. Lau, D. J. Barros, and J. M. Kahn, "Coherent detection in optical fiber systems," Optics express, vol. 16, no. 2, pp. 753-791, 2008.

[10] M. G. Taylor, "Coherent detection method using DSP for demodulation of signal and subsequent equalization of propagation impairments," IEEE Photonics Technology Letters, vol. 16, no. 2, pp. 674-676, 2004.

[11] N. Cvijetic, M.-F. Huang, E. Ip, Y. Shao, Y.-K. Huang, M. Cvijetic, and T. Wang, "1.92 Tb/s coherent DWDM-OFDMA-PON with no highspeed ONU-side electronics over 100km SSMF and 1: 64 passive split," Optics express, vol. 19, no. 24, pp. 24 540-24 545, 2011.

[12] N. Cvijetic, A. Tanaka, P. N. Ji, K. Sethuraman, S. Murakami, and T. Wang, "SDN and OpenFlow for dynamic flex-grid optical access and aggregation networks," Journal of Lightwave Technology, vol. 32, no. 4, pp. 864-870, 2014

[13] D. Lavery, R. Maher, D. S. Millar, B. C. Thomsen, P. Bayvel, and S. J. Savory, "Digital coherent receivers for long-reach optical access networks," Journal of Lightwave Technology, vol. 31, no. 4, pp. 609620,2013

[14] K. Cho, K. Tanaka, T. Sano, S. Jung, J. Chang, Y. Takushima, A. Agata, Y. Horiuchi, M. Suzuki, and Y. Chung, "Long-reach coherent WDM PON employing self-polarization-stabilization technique," Journal of Lightwave Technology, vol. 29, no. 4, pp. 456-462, 2011.
[15] S. Smolorz, E. Gottwald, H. Rohde, D. Smith, and A. Poustie, "Demonstration of a coherent UDWDM-PON with real-time processing," in $O p$ tical Fiber Communication Conference and Exposition (OFC/NFOEC), 2011 and the National Fiber Optic Engineers Conference, no. PDP-D4. Los Angeles, CA, USA: IEEE, 2011, pp. 1-3.

[16] A. Shahpari, R. Ferreira, V. Ribeiro, A. Sousa, S. Ziaie, A. Tavares, Z. Vujicic, F. P. Guiomar, J. D. Reis, A. N. Pinto et al., "Coherent ultra dense wavelength division multiplexing passive optical networks," Optical Fiber Technology, vol. 26, pp. 100-107, 2015.

[17] H. Rohde, S. Smolorz, E. Gottwald, and K. Kloppe, "Next generation optical access: $1 \mathrm{Gbit} / \mathrm{s}$ for everyone," in Optical Communication, 2009. ECOC'09. 35th European Conference on, no. paper 10.5.5. Vienna, Austria: IEEE, 2009, pp. 1-3.

[18] V. Sales, J. Segarra, V. Polo, J. C. Velásquez, and J. Prat, "UDWDMPON using low-cost coherent transceivers with limited tunability and heuristic DWA," Journal of Optical Communications and Networking, vol. 8 , no. 8, pp. 582-599, 2016

[19] C. Xie, P. J. Winzer, G. Raybon, A. H. Gnauck, B. Zhu, T. Geisler, and B. Edvold, "Colorless coherent receiver using $3 \times 3$ coupler hybrids and single-ended detection," Optics express, vol. 20, no. 2, pp. 1164-1171, 2012.

[20] S. Narikawa, H. Sanjoh, N. Sakurai, K. Kumozaki, and T. Imai, "Coherent WDM-PON using directly modulated local laser for simple heterodyne transceiver," in Optical Communication, 2005. ECOC 2005. 31st European Conference on, vol. 3. Glasgow: IET, 2005, pp. 449450.

[21] J. Segarra, V. Sales, J. Prat, and R. Pous, "A new flexible ONU design for UDWDM-PON with coherent transceivers and smart activation process," in Telecommunications Network Strategy and Planning Symposium (Networks), 2014 16th International. IEEE, 2014, pp. 1-5.

[22] "European Project COCONUT (FP7)." [Online]. Available: "http: //ict-coconut.eu"

[23] A. Rafel and N. Parkin, "Cost analysis of coherent-based TRx PON network," in Optical Communication (ECOC), 2015 European Conference on, no. paper P7.16. Valencia, Spain: IEEE, 2015, pp. 1-3.

[24] G. Vall-llosera, A. Rafel, N. Parkin, M. Angelou, D. Klonidis, I. Cano, M. Presi, G. Papastergiou, I. Tomkos, J. Prat et al., "COCONUT cost, power consumption and migration analysis: A route towards NG-PON3," in Transparent Optical Networks (ICTON), 2015 17th International Conference on, no. paper Mo.D2.1. Budapest,HU: IEEE, 2015, pp. $1-4$.

[25] I. Cano, J. Prat, J. Tabares, J. Velasquez, S. Ghasemi, V. Polo, G. Chu, M. Presi, E. Ciaramella, M. Rannello et al., "Field-Trial of Low-Cost Coherent UDWDM-PON with Real-Time Processing, $\lambda$-Monitoring and EPON Coexistence," in ECOC 2016; 42nd European Conference on Optical Communication; Proceedings of, no. paper M.1.E5. Dusseldorf, DE: VDE, 2016.

[26] I. N. Cano, A. Lerin, V. Polo, and J. Prat, "Direct phase modulation DFBs for cost-effective ONU transmitter in udWDM PONs," IEEE Photonics Technology Letters, vol. 26, no. 10, pp. 973-975, 2014.

[27] J. Prat, M. Angelou, C. Kazmierski, R. Pous, M. Presi, A. Rafel, G. Vall-Llosera, I. Tomkos, and E. Ciaramella, "Towards ultra-dense wavelength-to-the-user: The approach of the COCONUT project," in Transparent Optical Networks (ICTON), 2013 15th International Conference on, no. paper Tu.C3.2. IEEE, 2013, pp. 1-4.

[28] J. Segarra, V. Sales, V. Polo, and J. Prat, "Dimensioning OLT architectures for UDWDM-PONs employing coherent transceivers," in Transparent Optical Networks (ICTON), 2015 17th International Conference on, no. paper Mo.D2.5. Budapest,HU: IEEE, 2015.

[29] "Gigabit Capable Passive Optical Networks (G-PON)", ITU-T Series Recommendations G.984.

[30] E. Ciaramella, "Polarization-independent receivers for low-cost coherent OOK systems," IEEE Photonics Technology Letters, vol. 26, no. 6, pp. 548-551, 2014.

[31] M. Artiglia, M. Presi, F. Bottoni, M. Rannello, and E. Ciaramella, "Polarization-Independent Coherent Real-Time Analog Receiver for PON Access Systems," Journal of Lightwave Technology, vol. 34, no. 8, pp. 2027-2033, 2016.

[32] M. Rannello, M. Artiglia, M. Presi, and E. Ciaramella, " $10 \mathrm{~Gb} / \mathrm{s}$ longreach PON system based on directly modulated transmitters and simple polarization independent coherent receiver," Optics Express, vol. 25, no. 15, pp. $17841-17846,2017$.

[33] R. Corsini, M. Presi, M. Artiglia, and E. Ciaramella, "10-Gb/s longreach PON system with low-complexity dispersion-managed coherent receiver," IEEE Photonics Journal, vol. 7, no. 5, pp. 1-8, 2015. 
[34] G. Y. Chu, V. Polo, A. Lerín, J. Tabares, I. N. Cano, and J. Prat, "1.25$3.125 \mathrm{~Gb} / \mathrm{s}$ per user PON with RSOA as phase modulator for statistical wavelength ONU," Optics Communications, vol. 357, pp. 34-40, 2015.

[35] G. Y. Chu, V. Polo, A. Lerín, I. Cano, and J. Prat, "Optimizing reflective semiconductor optical amplifier as phase modulator for low cost colorless ONU with $3 \times 3$ homodyne detection," in Asia Communications and Photonics Conference, no. paper AF2B.5. Optical Society of America, 2014, pp. AF2B-5.

[36] J. Tabares, V. Polo, I. Cano, and J. Prat, "Automatic $\lambda$-control with offset compensation in DFB intradyne receiver for udWDM-PON," IEEE Photonics Technology Letters, vol. 27, no. 4, pp. 443-446, 2015.

[37] M. Artiglia, F. Bottoni, R. Corsini, M. Presi, M. Rannello, M. Valvo, and E. Ciaramella, " $4 \times 10 \mathrm{~Gb} / \mathrm{s}$ coherent WDM-PON system over $110 \mathrm{~km}$ single mode fibre and with $55 \mathrm{~dB}$ ODN power budget," in Optical Fiber Communications Conference and Exhibition (OFC), 2016, no. paper W2A-66. IEEE, 2016, pp. 1-3. 